NAisA' Contractor Report 181637

ICASE REPORT NO. $88-17$

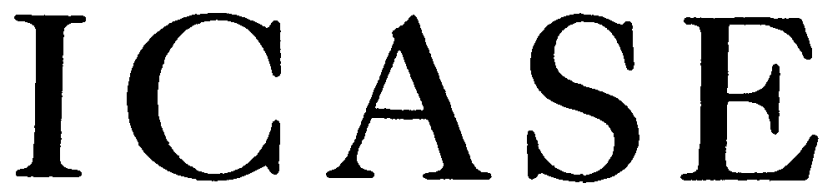

ADAPTIVE CONTROL OF A MANIPULATOR

WITH A FLEXIBLE LINK

(YASA-CR-181637) ACAETIVE CCAIECL OP A BAITELATOR DITE LFIEXIBLE LIXK Final

Eeport (BASA) $2 \mathrm{C}$ p CSCI $13 I$

N $88-18807$

$\begin{array}{ll}\text { Unclas } & \text { Unc32 } \\ 0128903\end{array}$

Y. P. Yang

J. S. Gibson

Contract No. NAS $1-18107$

February 1988

IUSTITUTE FOR COYPUTER APPLICATIOAS IN SCIEHCE AMD EMGIMBRIMG

MASA Langley Research Center, Bmpton, Virginia 23665

Operated by the Univeraities Space Research Association

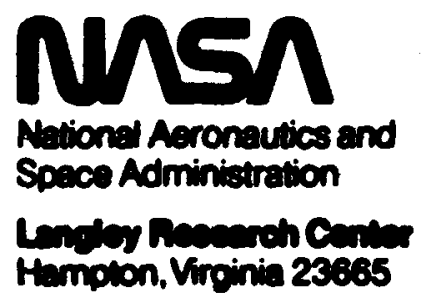




\title{
ADAPTIVE CONTROL OF A MANIPULATOR WITH A FLEXIBLE LINK
}

by

Y. P. Yang and J. S. Gibson

Department of Mechanical, Aerospace and Nuclear Engineering

University of California, Los Angeles 90024

\begin{abstract}
An adaptive controller for a manipulator with one rigid link and one flexible link is presented. The performance and robustness of the controller are demonstrated by numerical simulation results. In the simulations, the manipulator moves in a gravitational field and a finite element model represents the flexible link.
\end{abstract}

This research was supported by the National Aeronautics and Space Administration under NASA Contract No. NAS1-18107 while the second author was in residence at the Institute for Computer Applications in Science and Engineering (ICASE), NASA Langley Research Center, Hampton, VA 23665. Additional support was provided for both authors by AFOSR Grants 840309 and 870373. 


\section{Introduction}

There is extensive literature on adaptive control of robotic manipulators with rigid links [1-4]. Recently, researchers have begun to apply adaptive control to manipulators with flexible links. For a linear flexible link, Nelson, Miltra and Boie $[5,6]$ use an on-line parameter estimator to estimate an unknown payload and then compute periodic updates of optimal control gains that depend in a known explicit way on the payload mass. Chung and Leininger [7] applied adaptive pole placement to the six-degree-of-freedom JPL Standford arm, and in one simulation they included a static elastic deflection of the third link. Yuh [8] applied a discrete-time model reference adaptive controller to a single flexible link. The adaptive controller was designed for a rigid link disturbed by a process noise, which represented flexible modes, while the simulation model included the flexible modes. The adaptive controller in [8] appeared not to be able to suppress all oscillations about the final position of the link.

This paper presents a digital adaptive control scheme for a manipulator with one rigid link and one flexible link. The adaptive control algorithm is indirect; i.e., the control law at each sampling time is based on a prediction model of the plant whose time-varying parameters are estimated adaptively. This prediction model is linear and of sufficient dimension to reflect some but not all of the elastic degrees of freedom in the plant. Section 2 describes the manipulator model, in which the flexible link is represented by three finite elements, and Section 3 discusses the prediction model and parameter estimation. Section 4 presents the control law, which minimizes a weighted one-step-ahead quadratic criterion involving a reference model. Section 4 also discusses a continuous-time PD control loop that improves robustness and reduces control chattering in the closed-loop system produced by the adaptive controller.

Section 5 presents simulation results. In the simulations, the manipulator was modeled by the comprehensive nonlinear model described in Section 2, even though the adaptive algorithm is based on a linear prediction model. Because the order of the prediction model is smaller, by two modes, than the order of the manipulator (plant), the numerical results in Section 5 show that the adaptive controller is robust with respected to unmodeled higher-frequency modes. The simulations also demonstrate the effectiveness of the PD loop in reducing control chattering and the adaptive controller's ability to handle unknown payloads. 


\section{Manipulator Model}

Figure 2.1 shows the two-link manipulator to be controlled. The two joints centered at $o_{1}$ and $o_{2}$ are modeled as rigid discs. The first link is uniform, rigid and clamped to the first disc; the second link is a uniform Euler-Bernoulli beam, clamped to the second disc. The first disc is pinned at point $o_{1}$, which is fixed, and the second disc is pinned to the end of the first link at point $o_{2}$. At the other end of the second link is a payload, modeled as a point mass $M_{2}$. A control torque $\mathrm{u}_{1}$ acts on the first disc, and a control torque $\mathrm{u}_{2}$ acts between the second disc and the second link.

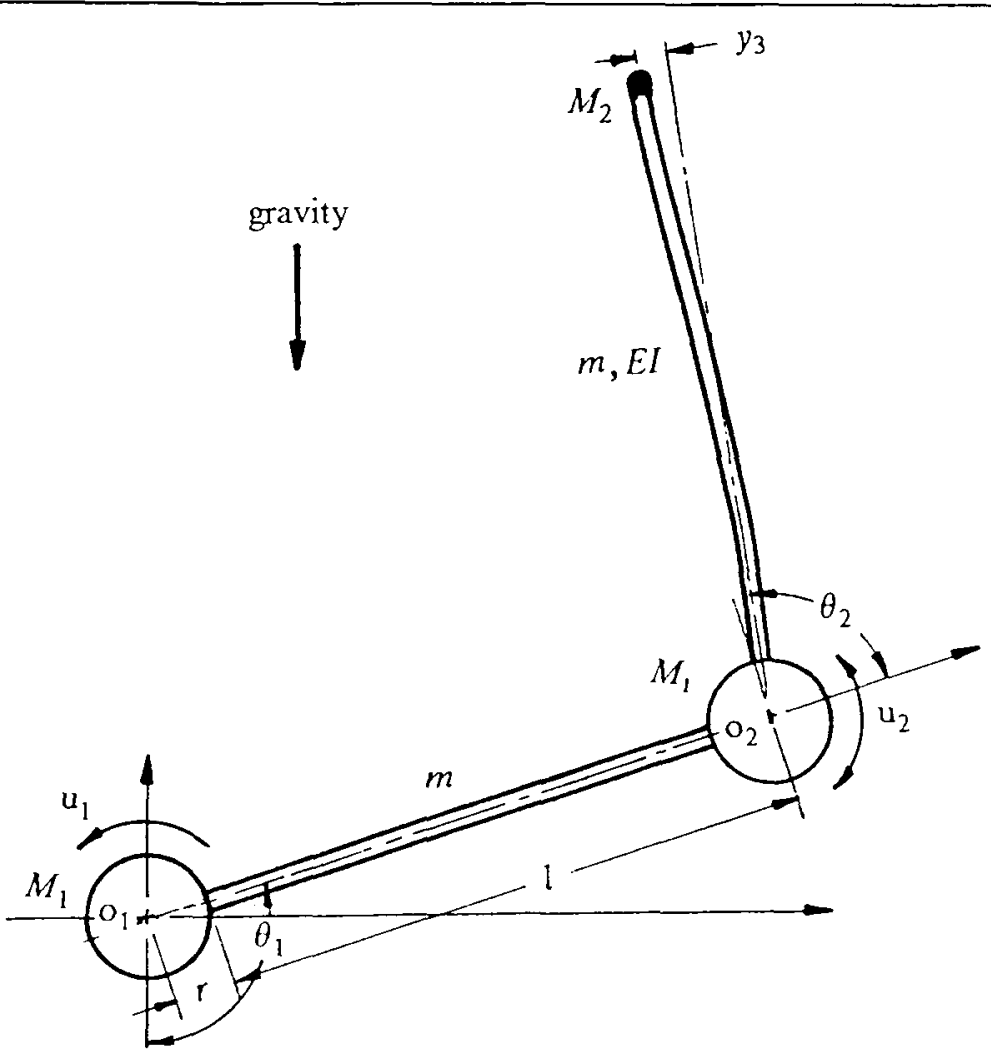

Figure 2.1 The Two-Link Manipulator

Table 2.1 System Parameters

$l=$ length of each link $=1.5 \mathrm{~m}$

$m=$ mass of each link $=1.2465 \mathrm{~kg}$

$E I$ of the flexible link $=3.99 \times 10^{6} \mathrm{Nm}^{2}$

$$
\begin{aligned}
& r=\text { radius of each joint }=.05 \mathrm{~m} \\
& M_{1}=\text { mass of each disc }=62.325 \mathrm{~g} \\
& M_{2}=\text { mass of payload }
\end{aligned}
$$


In our dynamic model of the manipulator, we include all nonlinearities that would be present if both links were rigid, and we model small (linear) transverse vibrations of the flexible link. We do not model axial vibrations of the flexible link, but we include the effect of the inertial axial load on the bending stiffness.

For simulating the response of the manipulator, we approximate the flexible link with three finite elements of equal length and we use cubic B-splines [9] as basis functions. This means that we have three elastic degrees of freedom, which we take to be the transverse elastic displacements of nodes 2,3 and 4 . (Node 1 is the end of link 2 attached to the second disc; node 4 is the end of link 2 to which the payload is attached.) With the two rigid-body degrees of freedom then, there are five degrees of freedom in our simulation model of the two-link manipulator.

For the finite element model of the manipulator, the generalized displacement vector is $\mathrm{q}=\left[\begin{array}{lllll}\theta_{1} & \theta_{2} & q_{3} & q_{4} & q_{5}\end{array}\right]^{\mathrm{T}}$ where $\theta_{1}$ and $\theta_{2}$ are the rigid-body angles and $q_{3}, q_{4}$ and $q_{5}$ are the transverse elastic displacements of nodes 2-4 on the second link. Lagrange's equations for the finite element model have the form

$$
\left[\mathbf{M}(\mathrm{q})+\mathbf{M}_{a}(\mathrm{q})\right] \ddot{\mathrm{q}}+\mathbf{D} \dot{\mathrm{q}}+\left[\mathbf{K}+\mathbf{K}_{a}(\mathrm{q}, \dot{\mathrm{q}})\right] \mathrm{q}+\mathrm{N}(\mathrm{q}, \dot{\mathrm{q}})=\mathbf{B u},
$$

where $\mathbf{M}(\mathrm{q})$ is a symmetric, positive definite mass matrix, $\mathbf{K}$ is the symmetric, nonnegative stiffness matrix due to the bending stiffness of the second link, $N(q, \dot{q})$ is a vector containing various gravity and inertial torques and $\mathbf{B}$ is a matrix containing 1 's and 0 's. The matrices $\mathbf{M}_{a}(q)$ and $\mathbf{K}_{a}(\mathrm{q}, \dot{\mathrm{q}})$ represent the effect of the inertial axial load on the stiffness of the flexible link; $\mathbf{K}_{a}(q, \dot{q})$ is symmetric but $\mathbf{M}_{a}(q)$ is not. In our model, the damping matrix $\mathbf{D}$ is equal to $10^{-5}$ times the part of the mass matrix that would correspond to the flexible link if $\theta_{1}$ and $\theta_{2}$ were held constant; this means that we model small proportional damping for the flexible link. A complete derivation of the equations of motion in given in [10].

Two observations about (2.1) that are very important for our purposes can be made from the detailed equations of motion in [10]. First, $q$ and $\dot{q}$ can be factored out of $N(q, \dot{q})$ in such a way that (2.1) can be written

$$
\mathbf{M}(t) \ddot{\mathbf{q}}+\mathbf{D}(t) \mathbf{q}+\mathbf{K}(t) \mathbf{q}=\mathbf{B u}
$$

where the matrices $\mathbf{M}(t), \mathbf{D}(t)$ and $\mathbf{K}(t)$ are polynomials in $\mathrm{q}(t), \dot{\mathrm{q}}(t), \cos \theta_{i}(t), \sin \theta_{i}(t)$ and $\left(\sin \theta_{i}(t)\right) / \theta_{i}(t)$. Second, for sufficiently small elastic vibration of the flexible link, no dominant terms in the matrices $\mathbf{M}(t), \mathbf{D}(t)$ and $\mathbf{K}(t)$ involve the elastic displacements $q_{3}, q_{4}$ and $q_{5}$. Hence the dominant terms in the coefficient matrices in (2.1) vary no faster than the rigid-body angles and angular velocities. 


\section{Prediction Model and Parameter Estimation}

Now we consider digital control of (2.1) and (2.2) by zero- order sample and hold; i.e., at the beginning of the $\mathrm{k}^{\text {th }}$ sampling interval $(\mathrm{k}=0,1,2, \ldots)$, we sample an output vector $\mathrm{y}(\mathrm{k})$ and apply a constant control vector $\mathrm{u}(\mathrm{k})$ for the duration of the $\mathrm{k}^{\text {th }}$ sampling interval. We take $\mathrm{y}=\left[\theta_{1} \theta_{2} y_{3}\right]^{\mathrm{T}}$ where $\theta_{1}$ and $\theta_{2}$ are the the rigid-body angles and $y_{3}=q_{5}$ is the transverse elastic deflection of the end of the flexible link that holds the payload.

According to standard linear system theory, an input/output model for (2.2) with digital input and digital linear output has the form of the ARMA model

$$
\mathrm{y}(\mathrm{k})+\sum_{\mathrm{i}=1}^{n_{a}} \mathrm{~A}_{\mathrm{i}}(\mathrm{k}) \mathrm{y}(\mathrm{k}-\mathrm{i})=\sum_{\mathrm{i}=1}^{n_{a}} \mathrm{~B}_{\mathrm{i}}(\mathrm{k}) \mathrm{u}(\mathrm{k}-\mathrm{i})
$$

where $A_{i}$ and $B_{i}$ are matrices of appropriate dimension and $n_{a}$ is an integer not greater than twice the dimension of $\mathrm{q}$ (i.e., $n_{a} \leq 10$ ). If the sampling rate is fast compared to the time rates of change of the dominant terms in the coefficient matrices in (2.2) (i.e., if the sampling rate is fast compared to the rigid-body angular velocities and accelerations), then the coefficient matrices in (3.1) can be considered to vary slowly. In this case, an adaptive parameter estimator should be able to track the coefficients in (3.1) and predict $y(k)$ from data taken through time $k-1$. Such prediction is the basis for the subsequent adaptive control algorithm.

In (3.1), the coefficients $A_{i}(k)$ may be full matrices, in which case $n_{a}$ is minimum, or they may be constrained to be diagonal. If the coefficient matrices in (2.2) were constant, the $A_{i}(k)^{\prime} s$ could be taken as scalar coefficients. We have found that our adaptive control scheme works best for the manipulator in this paper when the $\mathrm{A}_{\mathrm{i}}(\mathrm{k})^{\prime} s$ are diagonal with the second and third diagonal elements constrained to be equal in each $A_{i}(k)$; i.e., one independent ARMA model is used for the first output channel, which comes from the first link, and another independent ARMA model is used for the second and third output channels, which come from the second link. This is the kind of prediction model used in the simulation in Section 5.

For adaptive parameter estimation and output prediction, we use a standard recursive least squares algorithm [11, page 95] with a forgetting factor that varies with the magnitude of the prediction error as proposed in [12]. 


\section{Adaptive Control Algorithm}

\subsection{Control Law}

The ideal system output $\mathrm{y}_{*}$ is defined so that the error between the ideal output and a reference signal $y_{r}$ decays according to

$$
\left[\mathrm{y}_{*}(\mathrm{k}+1)-\mathrm{y}_{r}(\mathrm{k}+1)\right]+a_{e}(k)\left[\mathrm{y}_{*}(\mathrm{k})-\mathrm{y}_{r}(\mathrm{k})\right]=0
$$

where

$$
a_{e}(\mathrm{k})=\left(a_{0}-a_{f}\right) \gamma^{\mathrm{k}}+a_{f}
$$

with $a_{0}, a_{f}$ and $\gamma$ are positive scalars less than 1 . To make the true output $y(k)$ approximate the ideal performance, the control $u(k)$ is chosen at each step $k$ to minimize

$$
\begin{aligned}
J(\mathrm{k}) & =\left\|\hat{\mathrm{y}}(\mathrm{k}+1)-\mathrm{y}_{r}(\mathrm{k}+1)+a_{e}(\mathrm{k})\left[\mathrm{y}(\mathrm{k})-\mathrm{y}_{r}(\mathrm{k})\right]\right\|_{\mathbf{Q}}^{2}+\|\mathrm{u}(\mathrm{k})\|_{\mathbf{R}_{1}}^{2} \\
& +\|\mathrm{u}(\mathrm{k})-\mathrm{u}(\mathrm{k}-1)\|_{\mathbf{R}_{2}(\mathrm{k})}^{2}
\end{aligned}
$$

where $\mathbf{Q}$ is a nonnegative diagonal matrix, $\mathbf{R}_{1}$ and $\mathbf{R}_{2}(\mathrm{k})$ are positive definite diagonal matrices with

$$
\mathbf{R}_{2}(\mathrm{k})=\mathbf{R}_{20} \beta^{\mathrm{k}}
$$

for some nonnegative $\beta$ less than 1 , and the prediction $\hat{y}(k+1)$ is obtained from (3.1) with the least-square estimates $\hat{A}_{i}(k)$ and $\hat{B}_{i}(k)$ of the ARMA parameters. Since $R_{1}$ is positive definite, there is a unique $u(k)$ that minimizes $J(\mathrm{k})$, and this $\mathrm{u}(\mathrm{k})$ is a linear function of the histories of $\mathrm{y}, \mathrm{u}$, and $\mathrm{y}_{r}$. It is straightforward to write down the control law from (4.3). The gains in the control law vary with $a_{e}(\mathrm{k}), \mathbf{R}_{2}(\mathrm{k}), \hat{\mathrm{A}}_{\mathrm{i}}(\mathrm{k})$ and $\hat{\mathrm{B}}_{\mathrm{i}}(\mathrm{k})$.

This adaptive control algorithm is similar to model reference schemes discussed in [11, Sections 5.2 and 6.3]. An important difference between the control laws discussed there and the one here is that the error dynamics model in (4.1) and the penalty in (4.3) on the difference between successive control inputs vary with time. If the plant can be represented exactly by (3.1) with constant coefficients and if $\mathrm{y}$ and $\mathrm{u}$ have the same dimension, then stability results for the closed-loop system produced by our adaptive controller are similar to stability results in [11, Chapters 5 and 6$]$. In particular, if $\operatorname{rank}\left(B_{1}\right)=\operatorname{dim}(\mathbf{Q y})$ and $\mathbf{R}_{1}=\mathbf{R}_{2}=0$, then our adaptive controller reduces to a one-step-ahead model reference adaptive controller, and a sufficient condition for asymptotic stability is that all transmission zeros of the plant lie inside the open unit circle. 
Of course, (3.1) with constant coefficients cannot represent the manipulator in our problem exactly, but when the motion is linearized about an equilibrium position (and the control torques are perturbed about appropriate static torques), numerical results indicate that the square system that relates the torque perturbations to the perturbations in the rigid-body angles has all discrete-time transmission zeros on the unit circle when no open-loop damping is modeled and all transmission zeros strictly inside the unit circle when structural damping in the flexible link is modeled. We have demonstrated this numerically [10]; it is straightforward but tedious to write out the equation that we used. The analogous distributions of continuous-time transmission zeros for flexible structures with colocated sensors and actuators is well known.

Probably because a time-invariant linear ARMA model cannot represent the manipulator exactly and because we model very small structural damping, we have found that simple one-step-ahead adaptive control $\left(\mathbf{R}_{1}=\mathbf{R}_{2}=0\right)$ often produces an unstable system, even when we choose $\mathrm{y}=\left[\theta_{1} \theta_{2}\right]^{\mathrm{T}}$ to produce a minimum phase square plant. However, slight perturbations from this case have yielded effective stable controllers; i.e., $\mathbf{R}_{1}, \mathbf{R}_{2}(\mathrm{k})$ and the third diagonal element of $\mathbf{Q}$ are small. With the third output, we can improve the settling near equilibrium positions by placing a small penalty on elastic vibration. This requires either $\mathbf{R}_{1}$ or $\mathbf{R}_{2}(k)$ to be positive definite for there to exist a unique $u(k)$ to minimize $J(k)$. A positive definite $\mathbf{R}_{2}(\mathrm{k})$ serves a more important purpose, though. The plant zeros near the unit circle tend to produce chattering in the control, especially during the early large-angle motion when the prediction model is least accurate. We have been able to reduce such chattering substantially with small values of $\mathbf{R}_{2}(\mathrm{k})$. Near the final steady-state position, the motion is linear and the prediction model is more accurate, so that we do not need a positive $\mathbf{R}_{2}(k)$ to prevent chattering. Thus we allow $\mathbf{R}_{2}(k)$ to decay to zero asymptotically, thereby placing greater emphasis on near-steady-state output error in (4.3). We usually can eliminate the chattering by tuning $\mathbf{R}_{2}(\mathrm{k})$, but we do not yet have guidelines for this tuning.

We should note that our statements about stability of the closed-loop system consisting of the manipulator and the adaptive controller and about control chattering are based on two kinds of numerical results: using the nonlinear model of the manipulator to simulate the closed-loop response and on computing closed-loop eigenvalues for the linearized equations near an equilibrium position. The order (10) of the plant, the large nonlinearities in the plant and the fact that we need $a_{e}(k)$ and $\mathbf{R}_{2}(\mathrm{k})$ to vary with time in the control law, have prevented us so far from proving stability. 
While we have found that $\mathbf{Q}, \mathbf{R}_{1}$ and $\mathbf{R}_{2}(\mathrm{k})$ can be chosen to produce a stable closed-loop system for any final postion of the manipulator and any payload, the adaptive controllers often are not robust with respect to the choices of $\mathbf{Q}, \mathbf{R}_{1}$ and $\mathbf{R}_{2}(\mathrm{k})$. This lack of robustness appears to result from the near-nominimum phase characteristics of the plant. Robustness can be improved by inserting an inner continuous-time PD control loop with the (continuous-time) transfer function $\mathbf{T}_{\mathrm{PD}}(s)$ as shown in Figure 4.1. Such a control can shift the plant poles and zeros to give the adaptive controller an easier job. We have used decentralized PD loops at the individual joints to increase robustness, but we have had greater success with a PD loop designed according to [13] which provides at each joint a torque that is a linear function of rigid-body angular displacements and velocities at both joints. We give the PD gains in Section 5.

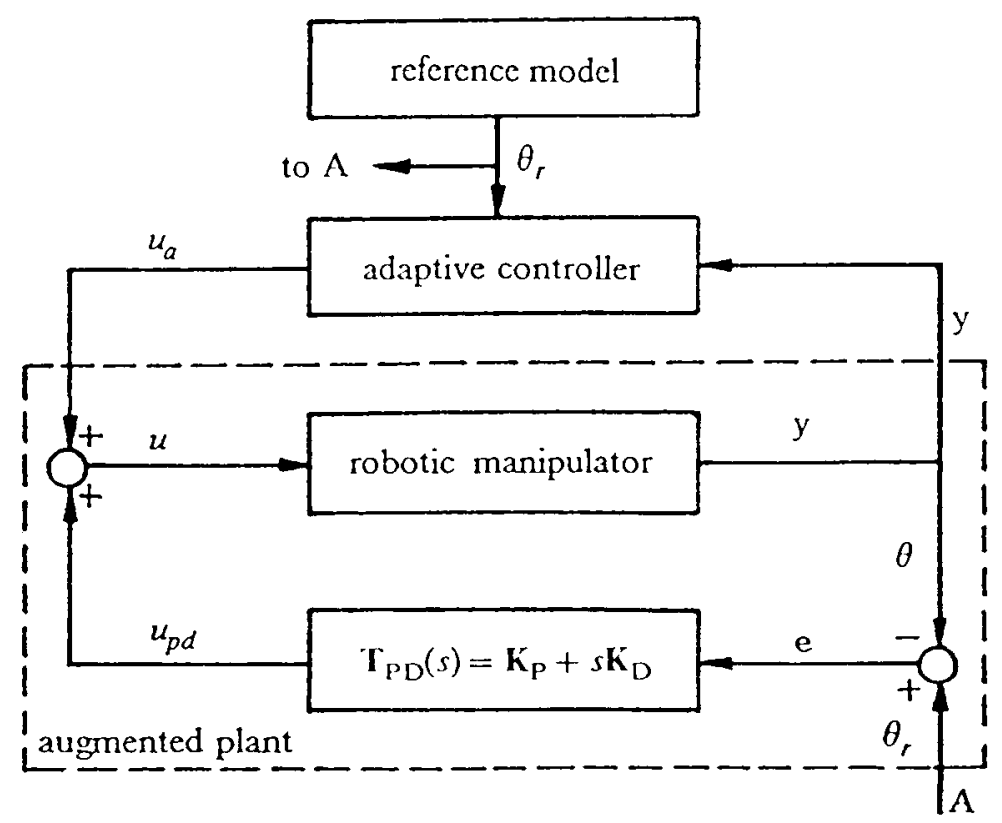

Figure 4.1 Control System

The the first two components of the reference signal $\mathrm{y}_{r}(\mathrm{k})--$ i.e., $\theta_{1 r}$ and $\theta_{2 r}-$ are computed off-line and are the outputs of a reference model that is chosen to ensure that $y_{r}(k)$ represents a reasonable response. In our scheme $\theta_{1 r}$ and $\theta_{2 r}$ are external inputs to the closed-loop system consisting of the manipulator and 
the adaptive controller, and the dynamics of the reference model that produces $\theta_{1 r}$ and $\theta_{2 r}$ do not affect the dynamics of the closed-loop system. Therefore, we will not discuss the details of this reference model except to say that we solved an optimal linear regulator problem for each of two uncoupled second-order oscillators to obtain two uncoupled linear reference models that produced the reference signals shown in Figures 5.1 - 5.3. See [10] for details. Different reference models should work.

The third component of $y_{r}(k)$ corresponds to the elastic tip deflection of the flexible link, which the control torques cannot drive to zero in the gravity field. We feed the measured tip deflection into a low pass filter and use the output of this filter as the third component of $y_{r}(k)$. This refenence signal is an estimate of the static tip deflection under gravity. During the large-angle motion of the manipulator, this probably is not a good estimate, but we take the third diagonal element of $\mathrm{Q}$ so small relative to the first two diagonal elements that the third component of $y_{r}(k)$ affects the control only near a steady-state postion, where the only remaining motion should be linear vibration about the static position.

For the low pass filter, we use the digital filter whose transfer function is

$$
T(z)=\frac{1-b}{z-b}
$$

where $\mathrm{b}=\exp \left(-\omega_{c} \mathrm{~h}\right)(\mathrm{h}=$ sampling time $=.01 \mathrm{sec})$ and the corresponding corner frequency $\omega_{c}$ is 4 Hz. This filter should attenuate the oscillations in the tip measurement, since the first natural frequency of the flexible link is $5.45 \mathrm{~Hz}$.

Adaptive control algorithms often use an initial learning period during which inputs and outputs vary only slightly from steady-state values to allow the parameter estimator to converge to initial parameter estimates for the prediction model before the controller begins to produce large changes in the state of the plant. We have found that a learning period is essential in the manipulator control problem here. Since the manipulator operates in a gravity field, nominal static torques are required to hold the manipulator near the initial position. Our control scheme assumes that the static torques are known within $10 \%$ for the case of zero payload. These torques (with $-10 \%$ error in our simulation) are taken as the control inputs during the first sampling interval $(.01 \mathrm{sec})$-- even when the manipulator has an unknown payload -- and it is the adaptive controller's job to hold the manipulator near the initial position for a learning period of at least 45 samples, after which the leaming period ends when the rigid-body angles are within $0.12 \mathrm{rad}$ of the initial values and predicted values of these angles are within $20 \%$ of the correct values. During the learning period, $\mathbf{R}_{2}(\mathrm{k})$ is set equal to a constant diagonal matrix and the magnitudes of the control torques are constrained 
not to exceed 1.5 times the magnitudes of initial torques. After the learning period, $\mathbf{R}_{2}(k)$ is reset to a smaller matrix and then decays according to (4.4). The larger $\mathbf{R}_{2}(k)$ and the torque constraint help prevent torques based on erroneous early parameter estimates from causing the manipulator to move significantly during the learning period.

\section{Simulation}

In the simulations reported here, the adaptive controller moves the manipulator from the horizontal position $\left(\theta_{1}=90^{\circ}, \theta_{2}=0^{\circ}\right)$ to the position $\theta_{1}=135^{\circ}, \theta_{2}=45^{\circ}$. (The motion is in a vertical plane, under gravity.) The initial elastic deflection is zero, and the final elastic deflection also is zero because the final position of the flexible link is vertical. (For final positions with nonzero static tip deflection, the estimate of this deflection produced by the filter in (4.5) can be used to correct the error in the final absolute tip position by small increments in $\theta_{1}$ and $\theta_{2}$.)

On each sampling interval, the nonlinear response of the manipulator was simulated on UCLA's IBM 3090 computer by solving the equations of motion in (2.1) with a fourth-order Runge-Kutta algorithm with variable step size $[14$, pages $83-84]$. The sampling rate is $100 \mathrm{~Hz}$.

The control parameters in (4.2)-(4.3) are

$$
\begin{aligned}
& \mathbf{Q}=\operatorname{diag}[30,20, .02] \quad \mathbf{R}_{1}=\operatorname{diag}\left[10^{-5}, 10^{-5}\right] \\
& \left.\begin{array}{l}
a_{0}=.98 \quad a_{f}=.98 \quad \gamma=1 \\
\mathbf{R}_{20}=\operatorname{diag}\left[2 \times 10^{-3}, 2 \times 10^{-2}\right] \quad \beta=1
\end{array}\right\} \text { during learning period } \\
& \left.\begin{array}{l}
a_{0}=.98 \quad a_{f}=.7 \quad \gamma=e^{-.007} \\
\mathbf{R}_{20}=\operatorname{diag}\left[2 \times 10^{-5}, 2 \times 10^{-4}\right] \quad \beta=.1^{.01}
\end{array}\right\} \text { after learning period }
\end{aligned}
$$

The order of the ARMA model used for prediction in the adaptive controller is $n_{a}=6$, even though the true plant order is 10. This reduced-order prediction model reflects our expectation that the second and third flexible modes in the simulation model are excited only slightly.

The continuous-time PD loop is based on the rigid-body equations of motion linearized about the final position. To demonstrate robustness with respect to plant uncertainties, the PD design is based on gravity torques and a rigid-body mass matrix that are $40 \%$ greater than their correct values for zero payload. The proportional and derivative gain matrices, designed according to [13], are 


$$
\mathbf{K}_{\mathrm{P}}=\left[\begin{array}{ll}
266793 & 82267 \\
82267 & 49114
\end{array}\right] \text { and } \quad \mathbf{K}_{\mathrm{D}}=\left[\begin{array}{cc}
896862 & 27234 \\
27234 & 13807
\end{array}\right]
$$

For the two-degree-of-freedom linear system on which these gains are based, the continuous-time PD controller produces a repeated pair of closed-loop eigenvalues at $-4 \pm 1.94 j$. See [10] for more detail.

In figures 5.1 and 5.2 , the payload $M_{2}$ is $20 \%$ of the mass of the flexible link. While the response of the manipulator is good in Figure 5.1, there is undesirable control chattering. The addition of the continuous-time PD loop substantially reduces the control chattering in Figure 5.2. Figure 5.3 shows the response for zero payload. The same adaptive control law was used for all three simulations, and the same inner PD loop was used for Figures 5.2 and 5.3. The tip oscillation and the control torques can be made smoother than in Figures 5.2 and 5.3 by adjusting the parameters in the control law after the PD loop is added, but using the same adaptive loop for all three simulations better demonstrates the adaptive capability.

The plots of the tip oscillations in all three simulations indicate that the first flexible mode is excited significantly, that the second mode is excited slightly during the early motion and that the third mode is negligible. Since the second mode can be seen in the early response, we conclude that the adaptive controller is robust with respect to this small unmodeled disturbance.

\section{References}

1. M. Tomizuka and R. Horowitz, "Model Reference Adaptive Control of Mechanical Manipulators", IFAC Adaptive Systems in Control and Signal Processing, San Francisco, 1983, pp.27-32.

2. S. Dubowsky and D. T. Desforges, "The Application of Model-Referenced Adaptive Control to Robotc Manipulators", Transactions of the ASME, Journal of Dynamics, Measurement, and Control, Vol. 101, Sept. 1979, pp.193-200.

3. K. Y. Lim and M. Eslami, "Adaptive Controller Designs for Robotic Manipulator Systems Yielding Reduced Cartesian Error", IEEE Transactions on Automatic Control, Vol. AC-32, No. 2, February 1987, pp.184-187.

4. M. Vukobratovic and N. Kircanski, "An Approach to Adaptive Control of Robotic Manipulators", Automatica, Vol. 21, No. 6, 1985, pp.639-647.

5. W. L. Nelson, D. Mitra and R. A. Boie, "End Point Sensing and Load-Adaptive Control of a Flexible Robot Arm", 1985 American Control Conference, June 1985, pp.1410-1415. 
6. W. L. Nelson and D. Mitra, "Load Estimation and Load Adaptive Optimal Control for a Flexible Robot Arm". 1986 IEEE International Conference on Robotics and Automation, pp.206-211.

7. C. H. Chung and G. G. Leininger, "Adaptive Self-Tuning Control of Manipulators in Task Coordinate System", Proceedings, International Conference on Robotics, Atlanta, Georgia, March 13-15, 1984, pp.608-612.

8. J. Yuh, "Application of Discrete-Time Model Reference Adaptive Control to a Flexible Single-Link Robot", Journal of Robotic Systems, 4(5), pp.621-630, October 1987.

9. M. H. Schultz, Spline Analysis, Englewood Cliffs, N. J., Prectice-Hall, 1973.

10. Y. P. Yang, Adaptive Control of Robotic Manipulators with Flexible Links, Ph.D. Dissertation, University of California, Los Angeles, 1987.

11. G. C. Goodwin and K. S. Sin, Adaptive Filtering, Prediction and Control, Prentice-Hall, Inc., Englewood Cliffs, New Jersey, 1984.

12. T. R. Fortescue, L. S. Kershenbaum and B. E. Ydstie, "Implementation of Self-Tuning Regulators with Variable Forgetting Factors", Automatica, Vol. 1, No. 6, 1981, pp. 831-835.

13. H. Seraji, "Design of Multivariable Controllers for Robot Manipulators", 1986 American Control Conference, Vol. 3, 1986, pp.1736-1741.

14. C. W. Gear, Numerical Solution of Ordinary and Partial Differential Equations, Prentice-Hall, Inc., Englewood Cliffs, New Jersey, 1971.

15. R. Fletcher, Practical Methods of Optimization, Vol. I, Unconstrained optimization, John Wiley \& Sons, New York, 1980. 

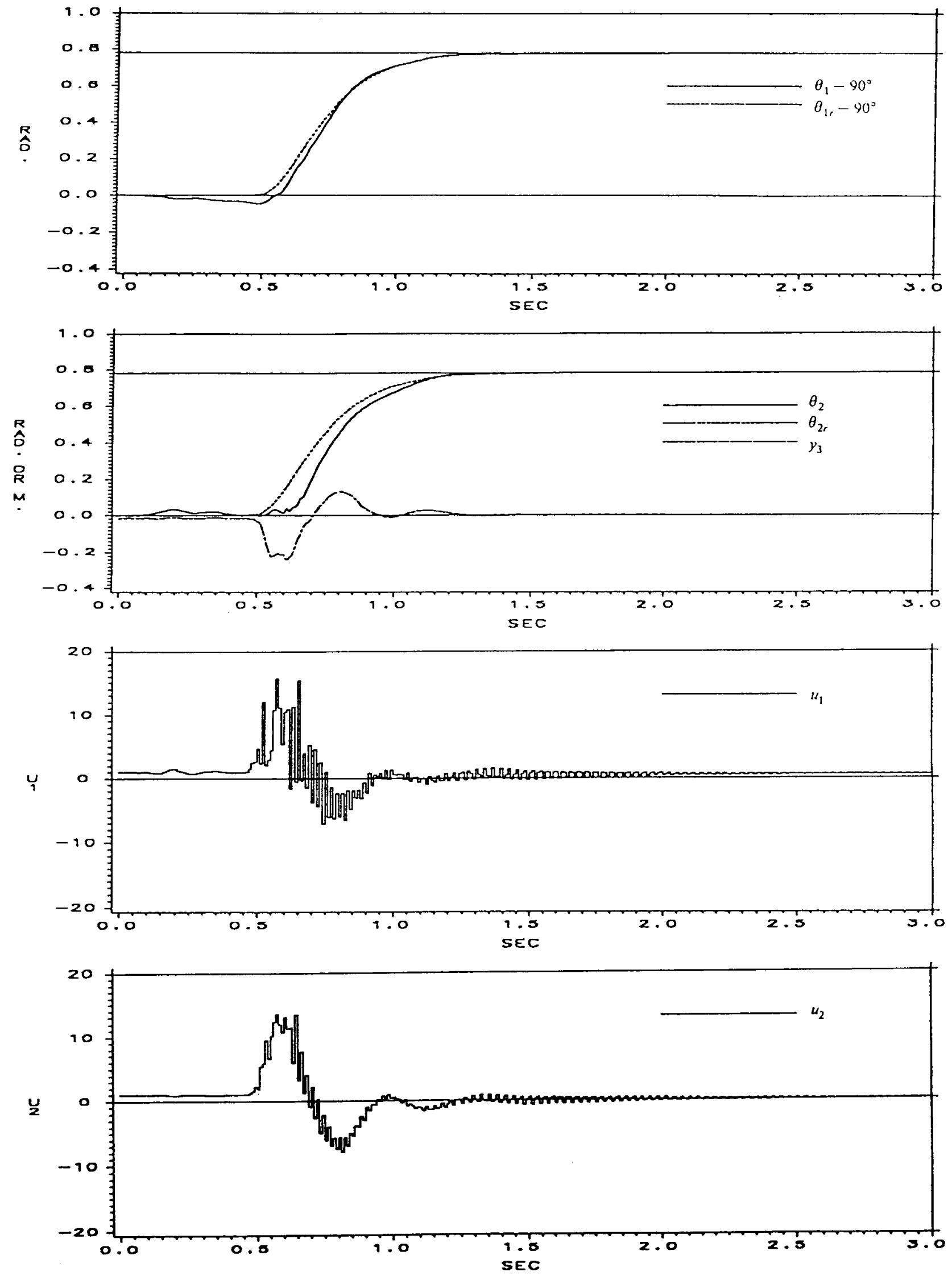

Figure $\underline{5.1}$ No PD loop; $M_{2}=.2 m$ 

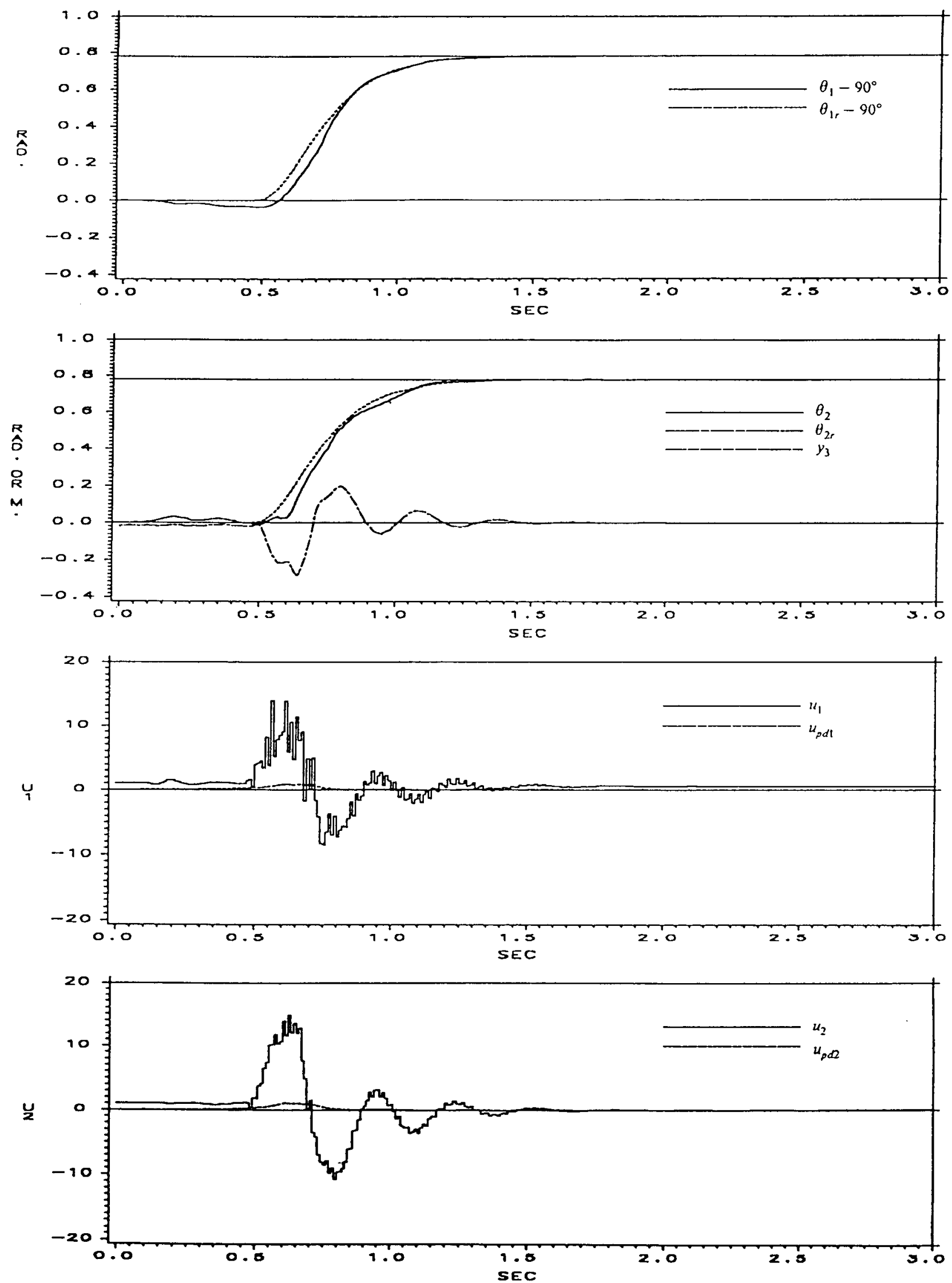

Figure 5.2 PD loop; $M_{2}=.2 \mathrm{~m}$ 

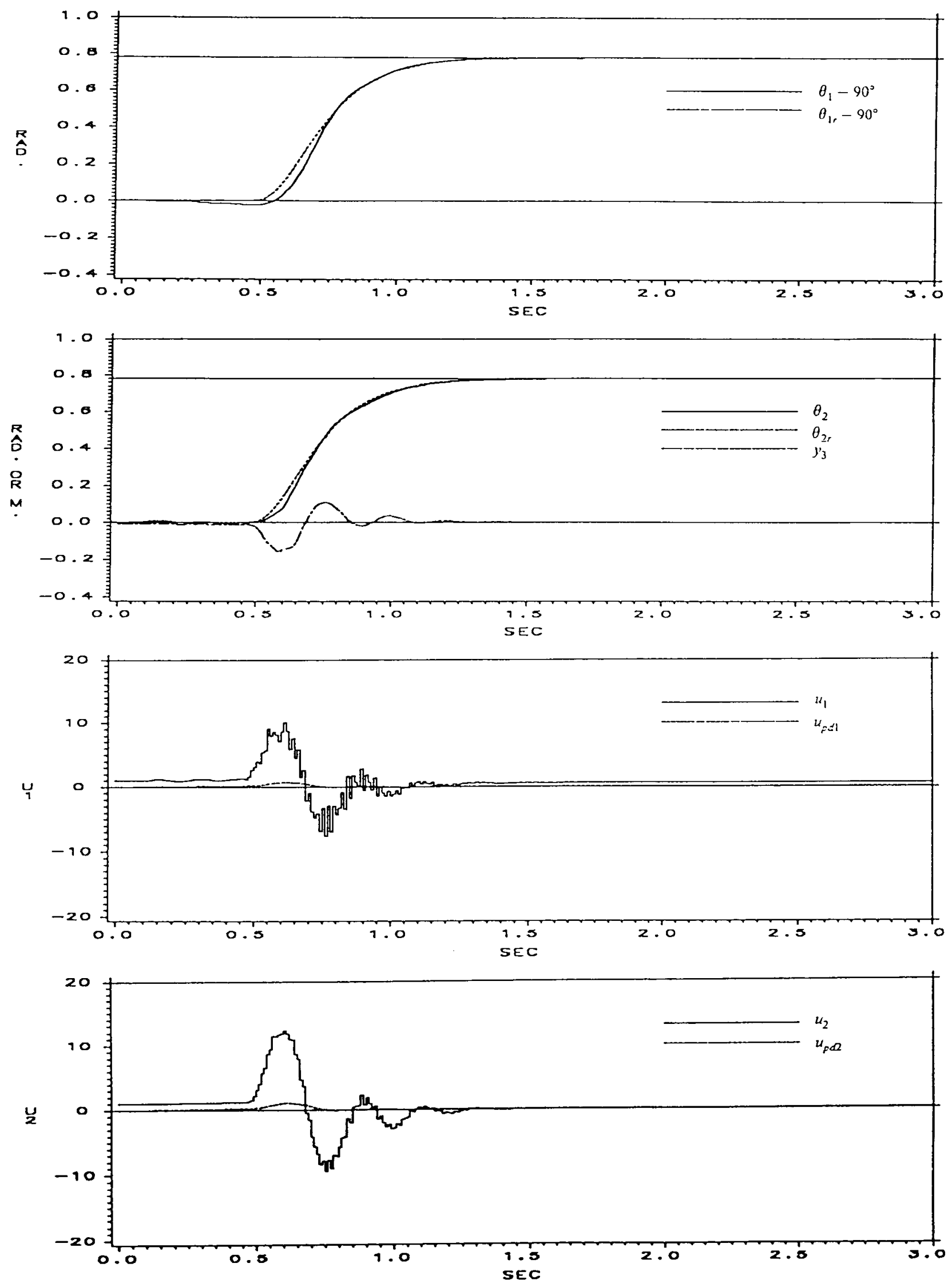

Figure 5.3 PD loop; $M_{2}=0$ 


\section{APPENDIX Accuracy of the Lincar AR.MA Model}

To show the accuracy of a linear time-varying AR.MA predictor for a manipulator moving in a large workspace, we applied our adaptive parameter estimator/predictor to an experiment performed in the Intelligent Systems Robotics Laboratory at the NASA Langley Research Center. The experimental data were obtained from the second joint of a UNIMATE 600 PUMA industrial robot with six degrees of freedom. The input torque $u$, by recording the motor voltage, and the rigid angle $\theta$ were measured. The sampling rate is $30 \mathrm{~Hz}$.

The parameters of the nonlinear model are first identified by the Levenberg-Marquardt method [15], which has been written into an IMSL subroutine ZXSSQ in FORTRAN language. The global trajectory is very hard to be matched with the nonlinear model

$$
\ddot{\theta}+c_{1} \dot{\theta}+c_{2} \dot{\theta}|\dot{\theta}|+c_{3} \sin \theta=c_{4} u
$$

where $c_{1} \dot{\theta}$ is a normalized viscous damping force, $c_{2} \dot{\theta}|\dot{\theta}|$ is a normalized quadratic friction force, $c_{3} \sin \theta$ is a normalized gravity force and $c_{4} u$ is a normalized input torque. These time invariant parameters in the time interval $[2.8,14]$ (seconds) are estimated as $c_{1}=5.59, c_{2}=11.52, \quad c_{3}=5.84, \quad c_{4}=11.78$. The expcrimental output and the predicted trajectory (i.e., the output of the model (A.1)) are shown in Figure A.1.

When a second-order linear ARMA model with single input and single output is selected in the form of

$$
\hat{\theta}(t)=-\sum_{i=1}^{2} \hat{a}_{i}(t) \theta(t-i)+\sum_{i=1}^{2} \hat{b}_{i}(t) u(t-i)
$$

the predicted output fits closely the experimental data, as shown in Figure A.2. The variation of parameters is shown in Figures A.3 and A.4. The parameters $\hat{a}_{i}$ and $\hat{b}_{i}$ are estimated by the recursive least-squares algorithm [11] with the forgetting factor at 1. It is somewhat surprising how well the linear ARMA model with the virtually constant parameters in Figures A.3 and A.4 predicts the output of the manipulator under the nonlinear gravity torque. 


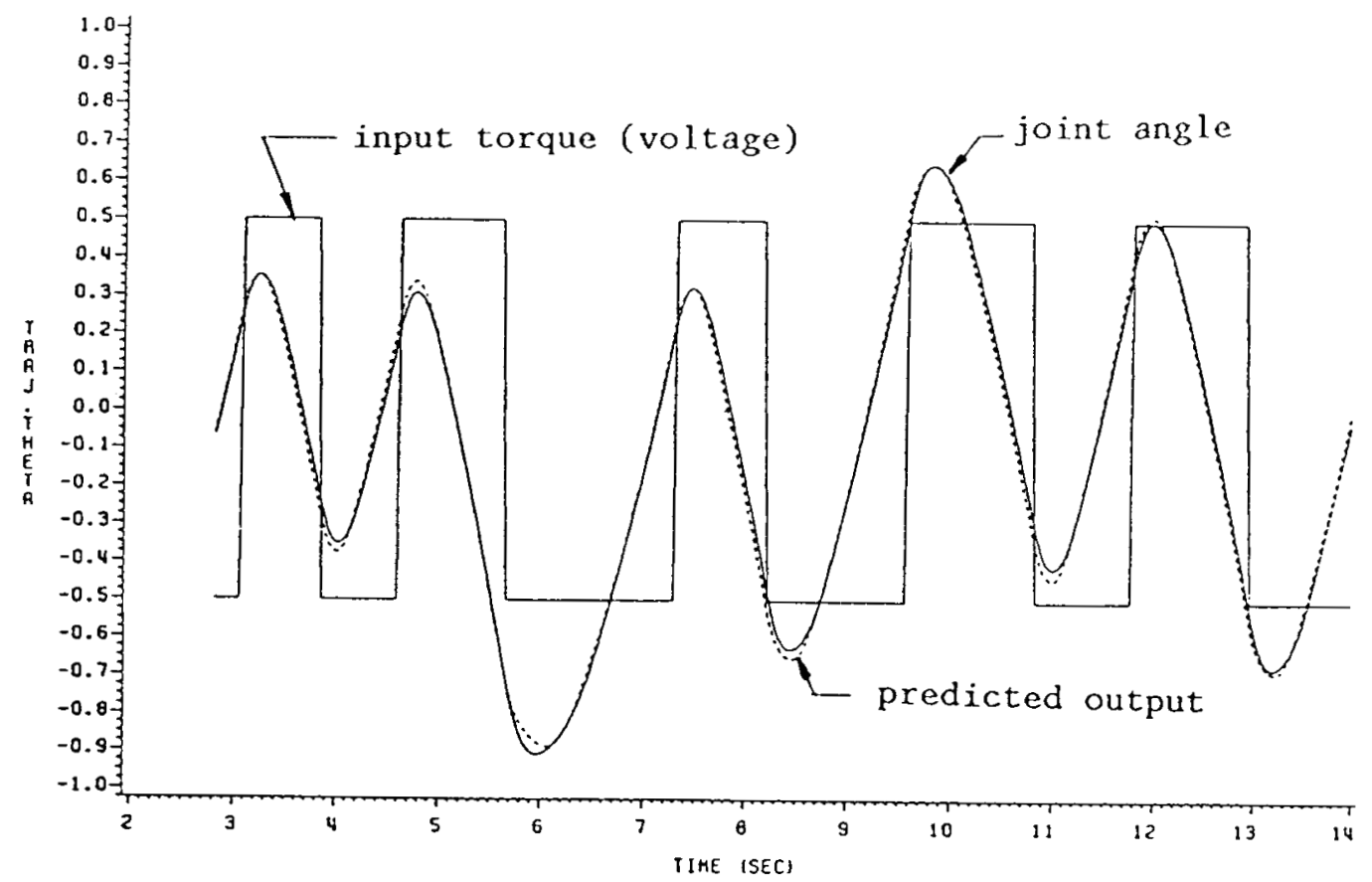

Figure A.1 Parameter Identification by Levenberg-Marquardt Method

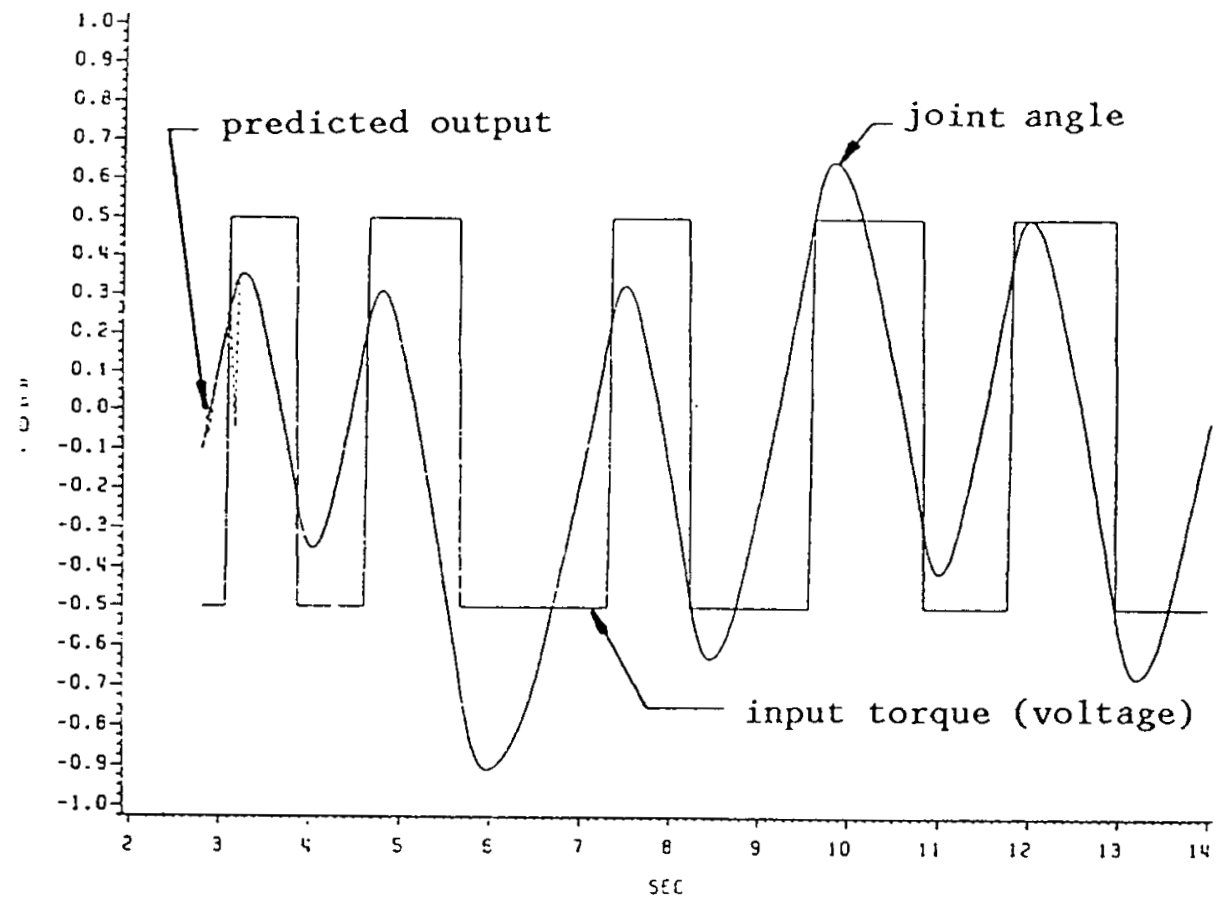

Figure A.2 Adaptive Parameter Identification with a Second-Order Linear ARMA Model 


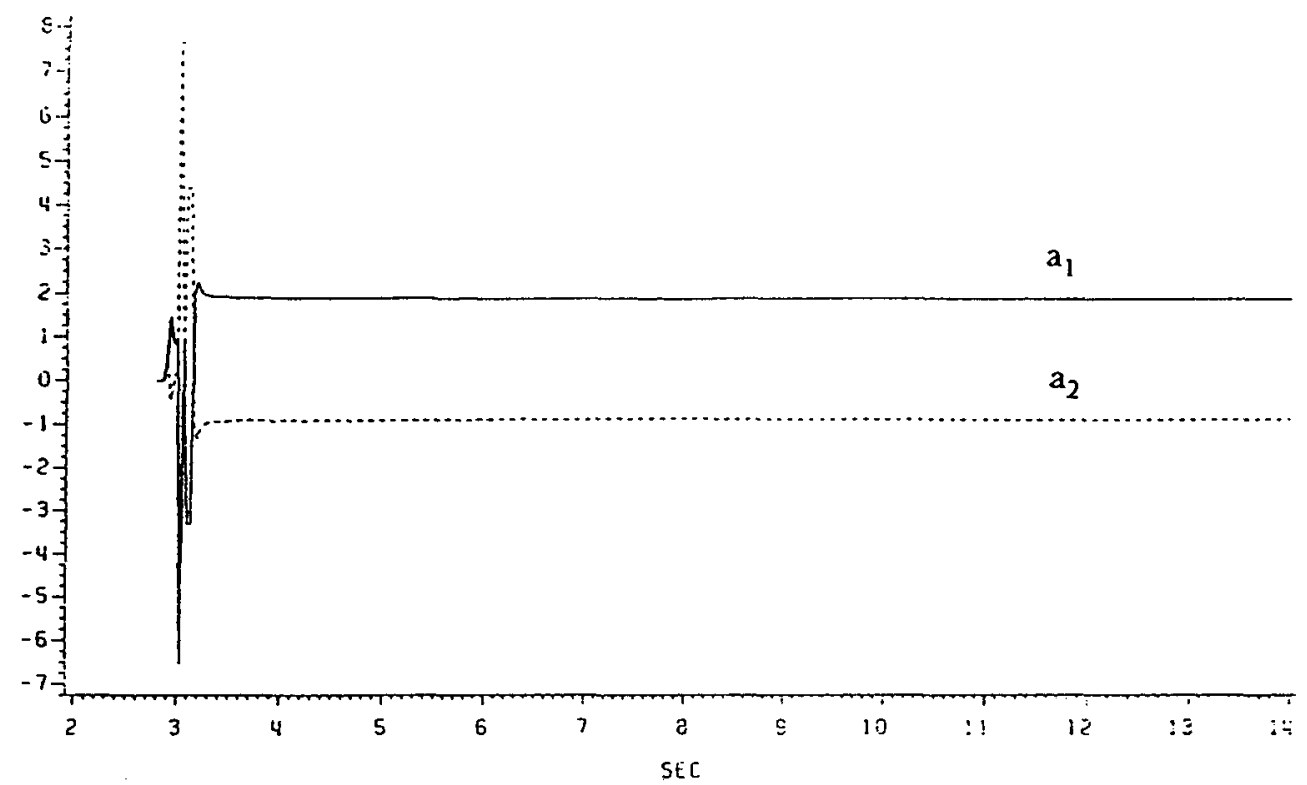

Figure A.3 Parameter Variation of the Second-Order Linear ARMA Model(a's)

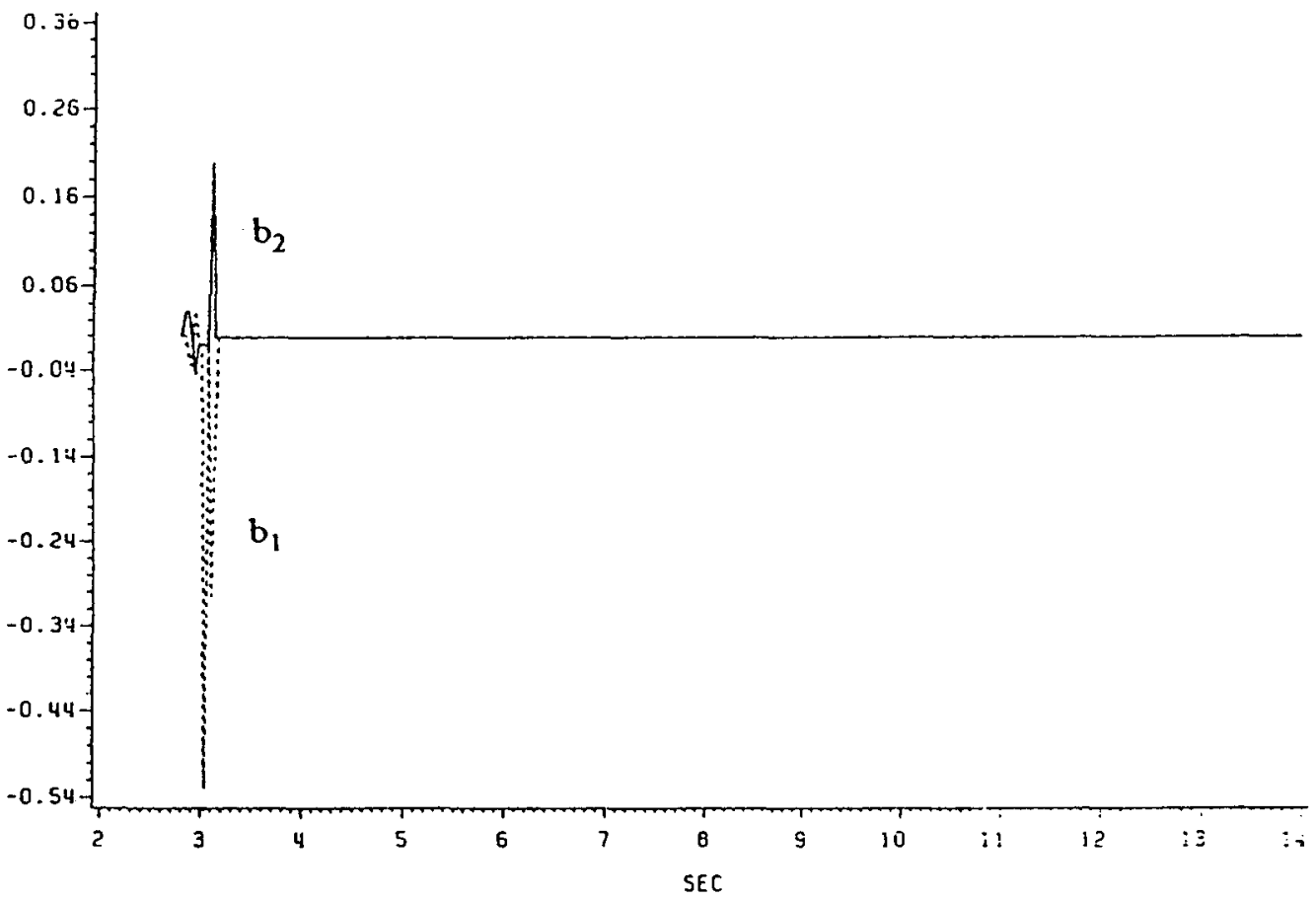

Figure A.4 Parameter Variation of the Second-Order Linear ARMA Model(b's) 


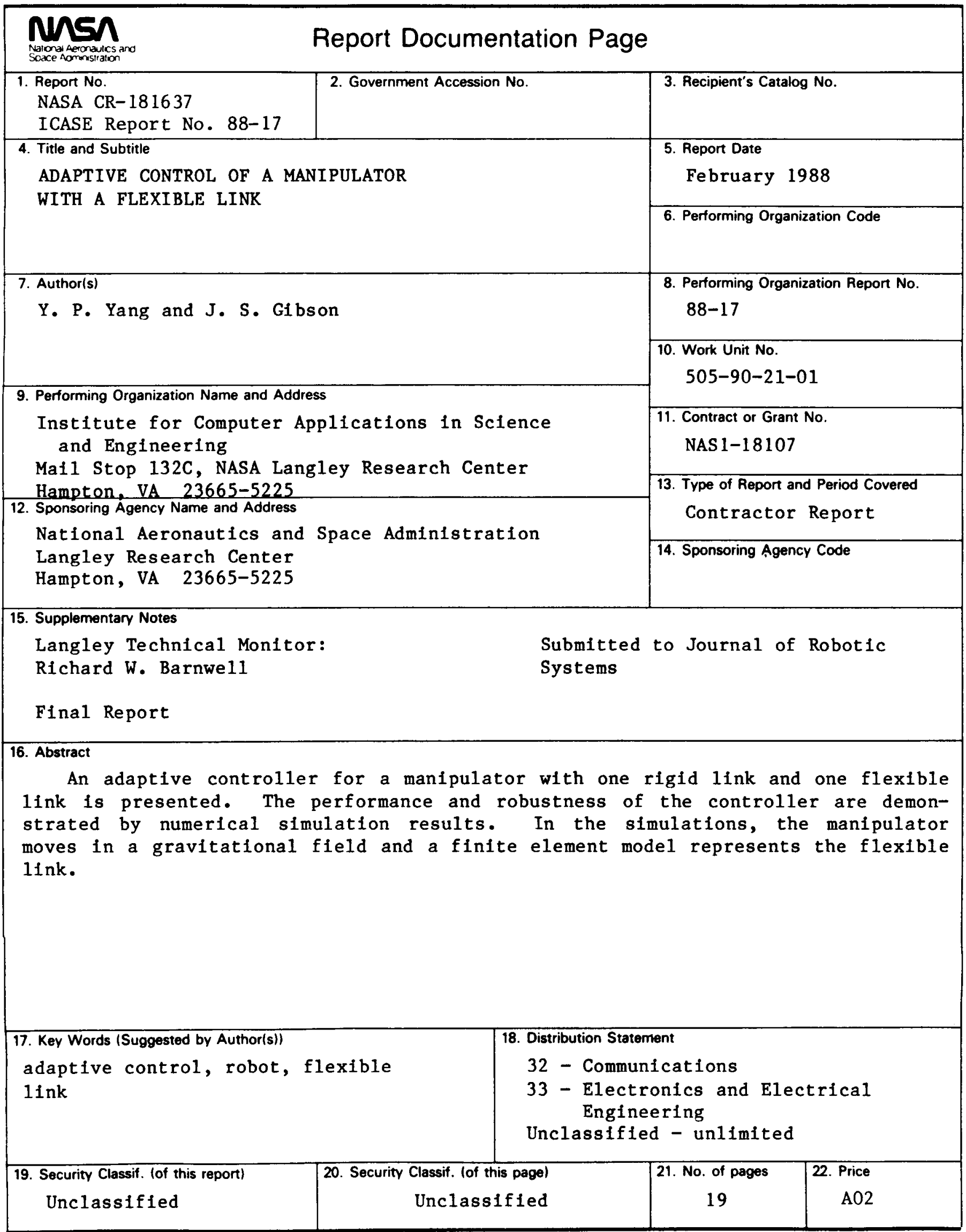

